
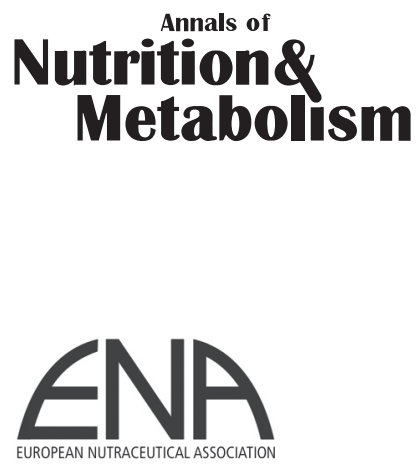

\title{
7th Annual Conference of the European Nutraceutical Association (ENA)
}

\section{Nutraceuticals and Women's Health}

March 12, 2011, Munich, Germany

\section{Abstracts and Poster}

\section{Guest Editors}

Manfred Lamprecht, Graz

Peter Prock, Basel 


\section{Annals of \\ Nutrition\& \\ Metabolism}

\section{Oral Presentations}

\section{1 \\ Supplementation with Nutraceuticals Improves Skin Health}

\author{
U. Heinrich \\ Dermatronnier, Institute for Experimental Dermatology, \\ University of Witten/Herdecke, Germany \\ E-mail: ulrike.heinrich@uni-wh.de
}

Summary: Numerous studies confirmed the efficacy of special dietary supplements (nutricosmetics). Antioxidants, vitamins and minerals, etc. are necessary for maintaining the health of skin and hair. They are, however, not always provided to a sufficient extent by our daily diet.

Therefore a supplementation with dietary supplements, at least temporarily, can be conducive to increase the photo-protection of the body or to improve skin characteristics. Avoiding undesirable side effects as well as toxicological risks must have the highest priority when it comes to the development of the products, especially if a long-term ingestion is intended. Dietary supplements should never be seen as a compensation for an unhealthy diet.

Introduction: For several years there has been a steady increase in the range of dietary supplements designed to maintain the health of skin and hair by guaranteeing an adequate supply of vitamins, minerals, antioxidants etc. For the substance category of carotenoids a function as antioxidants in lipophile systems has been established in recent years. As several studies confirm, carotenoids do not only protect algae and plants from intensive sunlight but they also function as photo-protective agents in humans [1,2]. Additionally, more recent studies show that carotenoids, often in combination with vitamins and minerals, contribute to the improvement of derma-physiological characteristics such as surface structure, density and thickness of the skin.

Improved efficacy can be achieved with the combination of carotenoids and vitamins, such as for instance alpha-tocopherol or vitamin C. This seems to be due to synergetic effects.

Furthermore, with regard to efficacy, the bioavailability of the agents plays a significant role. It is a known fact that carotenoids ingested in a denatured form (cooked) together with a lipid-containing carrier substance, hold a much higher level of bioavailability than raw vegetables [3]. There are also new technologies that increase bioavailability and thus also efficacy, for instance the coupling of the active agent, Lycopin (red coloring of tomatoes) with a lacto-protein [4]. For a useful supplementation of nutricosmetics one needs to pay attention that they have a good compatibility, high bioavailability, synergies (e.g. with vitamins) and that the intake is adjusted to the daily needs.
Methods and Results: Several studies with voluntary test persons have shown the positive influence of various nutricosmetics. Based on studies of our work group in the late 90s with various natural carotenoids, we primarily examined the photo-protective effect of this substance group. Our assessments proved that upon regular ingestion the sun protection effect of carotenoids was 3-4 times as high as the light protection naturally achieved in the body. With an additional vitamin E supplementation this effect could even be slightly improved. Thus, already in those days light-sensitive persons were recommended to supplement with carotenoids, even if only temporarily $[1,2]$.

Studies conducted at the Institute for Experimental Dermatology, at the University of Witten/Herdecke, also showed very positive effects in skin condition. The intake of a product with red clover isoflavones, vitamins and minerals resulted in a smoother skin surface relief. Over a 12- week test period roughness as well as scaling of the skin could be reduced. At the same time an improvement in the barrier function of the skin could be observed. This finding has proved to be particularly noteworthy since especially people with a very sensitive skin often suffer from a decreased skin barrier function. In such a case increased moisture release leads to increased dryness of the skin, which on very cold days causes a feeling of irritation and itching. After dietary supplementation the barrier function could be improved and the above described effects were clearly reduced.

Another very significant approach in the anti-aging concept is the improvement of elasticity. However, this particular aspect can only be influenced to a limited extent by external care. Nevertheless, studies with nutricosmetics show positive developments. The increase in skin density and thickness has a fortifying effect on the collagen structure of the skin, which in turn has a positive effect on its elasticity and vigor. The ingestion of a product with various natural carotenoids, combined with selenium and vitamin $\mathrm{E}$ over a period of 12 weeks resulted in a significant increase in skin density and thickness, which became apparent in an ultra-sound examination. At the same time, surface structure of the skin was positively influenced [5].

Other agents such as cocoa-polyphenols [6] or green tea extracts [7] also had positive effects on the density, surface structure and barrier function of the skin after a 12-week supplementation, thus also proving efficacy with regards to skin aging. In addition, studies with encapsulated vegetable and fruit juice concentrate improved skin microcirculation and ultrastructure of the skin [8].

All described studies were conducted according to strict, scientifically-oriented principles. Comparison groups treated without any active agents (placebo-groups) were included in the studies in order to exclude any other influences. Statistic analyses clearly showed the changes occurring within the respective groups and provided a comparison of the different groups.

The studies mentioned above all confirmed the lasting effects of nutricosmetics. Obviously our daily diet does not always guarantee a sufficient supply of antioxidants, vitamins and minerals despite a large supply of fresh fruits and vegetables. Thus, a combination of external care with quality products together with an internal supplementation with adequate nutricosmetics, in addition to a healthy diet

\begin{tabular}{ll}
\hline KARGER & (c) 2011 S. Karger AG, Basel \\
0250-6807/11/0581-0049\$38.00/0 \\
$\begin{array}{l}\text { Fax +4161306 1234 } \\
\begin{array}{l}\text { E-Mail karger@karger.ch } \\
\text { www.karger.com }\end{array}\end{array}$ & $\begin{array}{l}\text { Accessible online at: } \\
\text { www.karger.com/anm }\end{array}$
\end{tabular}


is an excellent basis to guarantee the optimal supply of nutrients for the skin.

However, fast results should not be expected. All the studies have shown that long-term adjustments are necessary, just as it is the case with nutrition, and only a regular ingestion of essential active agents in combination with adequate care for the respective skin-type will lead to the desired success.

\section{References:}

1. Heinrich U, Gärtner C, Wiebusch M, Eichler O, Sies H, Tronnier H, Stahl, W. Supplementation with $\beta$-Carotene or a similar amount of mixed carotenoids protects humans from UV-induced erythema: Journ Nutr. 2003; 133: 98-101.

2. Aust O, Stahl W, Sies H, Tronnier H, Heinrich U. Supplementation with tomato-based products increases Lycopene, Phytofluene and Phytoene levels in human serum and protects against UV light-induced erythema: Intern Journ Vitamin and Nutrition Research 2005;75:54-60.

3. Gärtner C, Stahl W, Sies H. Lycopene is more available from tomato paste than from fresh tomatoes: Am. J. Clin. Nutr. 1997, 6: 116-122.

4. Dreno B. New assessment methods applied to a patented Lacto-Lycopene, soy isoflavones and vitamin $\mathrm{C}$ formula in the correction of skin ageing: Les Nouvelles Dermatologiques, 22, 2003.

5. Heinrich U, Wiebusch M, Tronnier H, Sies H, Stahl W, Béjot M, Maurette JM. Antioxidant supplements improve parameters related to skin structure in humans: Skin Pharmacol Physiol 2006, 19: 224-231.

6. Heinrich U, Neukam K, Tronnier H, Sies H, Stahl W. Long-term ingestion of high flavonol cocoa provides photo-protection against UV induced erythema and improves skin condition in humans: J. Nutr. 2006; 136:1565-1569.

7. Heinrich U, Garbe B, Tronnier H, Stahl W, Moore C, Arnaud MMJ. Supplementation with Green Tea Extract improves skin physiological parameters: $17^{\text {th }}$ Congress of the European Academy of Dermatology and Venerology, Paris 2008, Poster Abstract 2008: FP 0324.

8. Heinrich U, Garbe B, Wiebusch M, Tronnier T. Supplementation with encapsulated vegetable and fruit concentrate improves microcirculation and ultrastructure in skin (Poster) Int. Congress of Experimental Biology; 2010, Anaheim, California, USA.

\section{2}

\section{Nutraceuticals in Pregnancy and Lactation}

\author{
E. Ogundipe \\ Imperial College, Chelsea \& Westminster Hospital, \\ London, UK \\ E-mail: e.ogundipe@imperial.ac.uk
}

Background: The health and wealth of a nation is dependent on an optimal diet and nutritional status of pregnant women as it lays the cornerstone of both physical and mental development in their children who become the future generation. It has been recognised that poor nutrient intake during pregnancy and lactation adversely affects the health of both the pregnant mother and her baby (1). Less affluent societies, especially in the developing world, are particularly at risk of maternal 'mis-nutrition' and infant malnutrition. The negative effect of infant malnutrition of a rapidly developing brain has now become a major concern of the United Nations World Food Programme (UN-WFP). It has been said that 'if children under two do not receive sufficient nutrition they will be sentenced to a lifetime of mental and physical limitations. We now have what I call the burden of knowledge and WFP is looking for ways to ensure we prioritize those under twos, the most vulnerable of all in the world' (Josette Shaaron Executive Director's report to the Board of UN-WFP; June 2010).

Mismatched maternal dietary and nutritional choices ('mis-nutrition') during pregnancy and lactation can have a very significant impact on fetal and neonatal brain development. Human and animal studies have shown that malnutrition has irreversible effects on brain size and function (2). Approximately $70 \%$ of brain development occurs before birth and nearly all the rest while the baby is breast-feeding so during this phase of the child's life an adequate maternal nutrition is mandatory for normal brain development (3). The effect of maternal nutrition becomes even more profoundly felt after preterm birth as the energy and nutrient supply often becomes even more sub-optimal post-natally with adverse consequences on the rapidly developing infant brain. One fundamental factor is the varying effect of timing of when supplements are taken on development especiallythe brain (4). For example, this may mean that an enhancing nutrient in early pregnancy may become toxic if taken in later pregnancy. Optimal maternal nutrition in pregnancy and lactation directly impacts not only on maternal health and well being, pregnancy outcome, pregnancy complications ante- and post-natally and mental health but also on their infants' health outcomes and brain development. Nurse (5) stated that predicted brain disorders will surpass even cardiovascular disease in the next few decades with an estimated health bill in the European union of over 386 billion Euros by 2020 and this potential explosion in mental health disorders may have the seeds sown not only in personal early life-style choices, but also in the dietary and nutritional habits of pregnant and lactating women. The most well known nutritional supplements regularly offered or taken in pregnancy include folic acid, iron, iodine, zinc, selenium, magnesium, vitamin D and also calcium especially in the lactating phase (6). The role of other vitamins and trace elements such as B12, vitamin A, E, K, copper, manganese are more variable and their roles less well documented in recent papers. More recently, there has been recognition of the importance of long chain polyunsaturated fatty acids (LCPUFAs) such as Arachidonic acid (AA), an omega-6 fatty acid and Docosahexaenoic acid (DHA), an omega-3 fatty acid obtained mainly from fish oil and other animal sources. These LCPUFAs have been shown to be essential for infant brain growth and development and to also significantly impact on pregnant and lactating mothers' health $(2,3)$.

The composition and requirements of nutraceuticals, with regards to both the macro- and micronutrient components in pregnancy and lactation is likely to vary between developed and developing countries, and there are recent reports $(7,8)$ that have examined the impact of nutraceuticals such as micronutrient supplementation during pregnancy and lactation on maternal and child health outcomes. Shrimpton et al (9) of the United Nations Multiple Micronutrient Preparation (UNIMMAP) studies in Asia and Africa, showed no advantage of the multiple micronutrient supplement (MMS) used over folic acid and iron supplements alone and the Cochrane review by Haider and Bhutta in 2009 (15) also found no advantage. Worryingly, they showed a statistically significant increase in neonatal mortality with data from 6 of the 7 countries (OR 1.46; 95\% confidence interval 1.09-1.95). Hyppönen and Boucher (10) also report a re-emergence of vitamin D deficiency in the developed world. Folic acid, probably the most well known supplement in pre- and during pregnancy is linked with increased congenital malformations when deficient. A Cochrane review by De-Regil et al (11) showed a reduction in neural tube and cardiac defects with supplementation. Iron supplementation will be discussed elsewhere. 


\section{References:}

1. Black RE et al. Maternal and Child undernutrition: global and regional exposuresand health consequences. Lancet 2008; 371: 243-260.

2. Crawford M.A., Golfetto I, Bistanis D, Ghebremeskel K, Min Y, Moodley T, Poston L, Phylactos A, Cunnane S, Schmidt W. (2003) Arachidonic and Docosahexaenoic Acids in Protection Against Central Nervous System Damage in Preterm Infants. Lipids 38 (4), 303-315.

3. Crawford, M.A., Doyle, W., Leaf, A., Leighfield, M., Ghebremeskel K. \& Phylactos, A. (1993) Nutrition and Neurodevelopmental Disorders. Nutr. and Health 9: 81-97.

4. Georgieff MK. Nutrition and the developing brain: nutrient priorities and measurement. Am J Clin Nutr 2007; 85: 614S-620S.

5. Nurse J. Brain disorders have over taken all other burdens of ill health in the EU costing $€ 386 \mathrm{~B}$ DoH Mental Health Division: www.westminsterforumprojects.co.uk/dietandhealthforum/slides/JoNurse.pdf.

6. UNICEF/WHO/UN 1999. Composition of a multi-micronutrient supplement to be used in pilot programmes among pregnant women in developing countries.

7. Osrin D et al. Effects of antenatal multiple micronutrient supplementation on birthweight and gestational duration in Nepal: double blind randomised controlled trial. Lancet 2005; 365:955-2.

8. Shrimpton et al. Multiple micronutrient supplementation during pregnancy in developing- country settings: Policy and program implications of the results of a meta-analysis. Food and Nutrition Bulletin 2009; 30: S556-S573.

9. Haider BA, Bhutta ZA. Multiple-micronutrient supplementation for women during pregnancy. Cochrane Database of Systemic Reviews 2006; Issue 4.: 10.1002/14651858.CD0044905.pub2.

10. De-Regil LM et al. Effects and safety of periconceptional folate supplementation for preventing birth defects. Cochrane Database of Systematic Reviews 2020, DOI:10.1002/14651858.CD007959.pub2.

11. Hyppönen E, Boucher BJ. Avoidance of vitamin D deficiency in pregnancy in the United Kingdom: a case for a unified approach in National policy. Br J Nutr 2010; 104: 309-314.

\section{3}

\section{Isoflavones and Female Health}

\author{
J.C. Huber \\ Department for Gynecological Endocrinology and \\ Reproductive Medicine, Medical University Vienna, \\ Vienna, Austria \\ E-mail: johannes.huber@meduniwien.ac.at
}

Beside the agonistic effect of Isoflavones on estradiol receptor beta, associated with a lot of beneficial effect, Isoflavones modulate the endogenous metabolism of estradiol and progesterone.

These natural hormones, produced predominatly in the ovaries or replaced by hormone therapy are hydroxylated in different position.

4 - and 16 hydroxyestron have a strong microgenic and angiogenic effect, important for human reproduction but a burden for the female body.

2-hydroxyestron has a more anti-angiogenic effect. Isoflavones modulates this hydroxylation, suppressing 4- and 16-hydroxy-estron and stimulating the hydroxylation in position 2 .

Further on 4-and 16-hydroxyestron generate free radicals in semichinons on the DNA which is also a burden for the female body.

Isoflavones are enzyme modulator and are therefore key player for female health. Beside them good microbiome are also important for the role of isoflavones and should be discussed shortly.

\section{References:}

Tempfer CB, Froese G, Heinze G, Bentz EK, Hefler LA, Huber JC. Side effects of phytoestrogens: a meta-analysis of randomized trials. Am J Med. 2009 Oct;122(10):939-46.e9. Review.

Tempfer CB, Bentz EK, Leodolter S, Tscherne G, Reuss F, Cross HS, Huber JC. Phytoestrogens in clinical practice: a review of the literature. Fertil Steril. 2007 Jun;87(6):1243-9. Epub 2007 May 9. Review.

\section{4 \\ Iron Deficiency and Iron Substitution in Women of Reproductive Age}

\section{A. Krafft}

Division of Obstetrics, Department of Obstetrics and Gynecology, University Hospital Zurich, Zurich, Switzerland E-mail: alexander.krafft@usz.ch

In recent years in obstetrics and gynecology but also in general medicine we observe a growing awareness of iron deficiency and therefore iron substitution in women of reproductive age.

Iron deficiency is the most common nutrient deficiency in the world. According to the WHO's World Health Report 2002, as many as $4-5$ billion people, or $66-80 \%$ of the world's population, may be iron deficient, while over $30 \%$ of the world's population are anemic, mainly due to iron deficiency [1]. With a focus on industrialized countries the problem of (iron deficiency) anemia is of a smaller magnitude, but iron deficiency is considered to be one of the main deficiency disorders affecting large fractions of the population, particularly menstruating and pregnant women. Women, especially adolescents consuming low-energy diets and vegetarians are at higher risk of developing iron deficiency. Depending on the reference cited prevalence of iron deficiency in Europe ranges from 4 up to $33 \%$ [2].

Although there is only little evidence that depleted iron stores have any severe adverse consequence, it does indicate that iron nutrition is borderline, since any further reduction in body iron stores (for instance due to heavy uterine bleeding or pregnancy) is associated with a decrease in the level of functional compounds such as hemoglobin. Once anemia develops, morbidity is increasing, especially in pregnancy and postpartum. Besides, the negative consequences of iron deficiency and anemia on cognitive and physical performance are of major concern. There is evidence that non-anemic women with unexplained fatigue may benefit from iron supplementation, especially if iron stores are low or borderline [3]. Another matter not of common knowledge is that a disturbance in iron transport, metabolism and storage might play an important role in the pathophysiology of restless legs syndrome. Several studies support the hypothesis, that a relative iron deficiency in the central nervous system can lead to a dopamine dysfunction and consequently cause restless legs syndrome [4].

Furthermore iron is one of the micronutrients of importance during the periconceptional period. Besides its role as an integral part of hemoglobin it is also essential in nucleic acid metabolism, enzyme systems, and intracellular electron transport. There is evidence that consumption of iron supplements may decrease the risk of ovulatory infertility [5].

In most cases iron deficiency can be measured quite precisely using serum ferritin. To exclude falsely elevated ferritin values, 
C-reactive protein is measured simultaneously ruling out inflammation. Whereas it is widely accepted that iron stores are depleted, if ferritin values are below $15 \mathrm{ug} / \mathrm{L}$, there is considerable debate on the ferritin value which reflects sufficient iron stores and, therefore, which value warrants iron supplementation.

Assuming iron deficiency being a preventable state a first step might be information about appropriate dietary habits, iron supplementation of groups at high risk (such as pregnant women) and iron fortification of foods (specifically oriented toward certain physiological groups such as children). Next step is individual iron supplementation. The most commonly prescribed treatment consists of oral administration of iron as ferrous sulfate [2]. However, oral administration of ferrous sulfate causes some well known side effects, including gastrointestinal discomfort, nausea, vomiting, diarrhea and constipation. Due to the often poor compliance, there is a constant need for treatment alternatives. One low-cost option could be lactoferrin. It is a naturally existing iron-binding multifunctional protein and is present at high concentrations in human milk and in the milk of other mammals. Another alternative to be found on the market is ferric polymaltose, which has a considerably lower adverse event rate than conventional ferrous sulfate preparations. Unfortunately, there is still ongoing debate on its bioavailability and efficacy [6].

If large amounts of iron need to be administered quickly and without considerable adverse events an elegant option is intravenous application of iron sucrose or its successor iron carboxymaltose. For both preparations efficacy and safety have been shown in numerous studies from different specialties [7, 8]. The main draw back is the higher therapy cost compared to oral iron substitution which has been shown to be efficient if tolerated by the patient in terms of duration and side effects of the treatment. As for most therapy schemes, a stepwise approach is the key to successful treatment of iron deficiency.

\section{References:}

1. Quantifying selected major risks to health, in The World health report: 2002: Reducing risks, promoting healthy life. 2002, World Health Organization: Geneva. p. 52-54.

2. Hercberg, S., P. Preziosi, and P. Galan, Iron deficiency in Europe. Public Health Nutr, 2001. 4(2B): p. 537-45.

3. Verdon, F., B. Burnand, C.L. Stubi, et al., Iron supplementation for unexplained fatigue in non-anaemic women: double blind randomised placebo controlled trial. Bmj, 2003. 326(7399): p. 1124.

4. Allen, R.P., P.B. Barker, F. Wehrl, et al., MRI measurement of brain iron in patients with restless legs syndrome. Neurology, 2001. 56(2): p. 263-5.

5. Cetin, I., C. Berti, and S. Calabrese, Role of micronutrients in the periconceptional period. Hum Reprod Update, 2010. 16(1): p. 80-95.

6. Nielsen, P., E.E. Gabbe, R. Fischer, et al., Bioavailability of iron from oral ferric polymaltose in humans. Arzneimittelforschung, 1994. 44(6): $\mathrm{p}$. $743-8$.

7. Perewusnyk, G., R. Huch, A. Huch, et al., Parenteral iron therapy in obstetrics: 8 years experience with iron-sucrose complex. Br J Nutr, 2002. 88(1): p. 3-10.

8. Bailie, G.R., Efficacy and safety of ferric carboxymaltose in correcting iron-deficiency anemia: a review of randomized controlled trials across different indications. Arzneimittelforschung, 2010. 60(6a): p. 386-98.

\section{Posters}

\section{5 \\ Homocysteine Metabilism and Oxidative Status in Healthy Heavy Smokers: Effects of Nutraceutical Supplementation}

\author{
F. Bamonti', C. Novembrino', R. De Giuseppe ${ }^{2}$, F. de Liso², \\ L.Vigna ${ }^{3}$, M. Pellegatta ${ }^{3}$, G. Cighetti ${ }^{4}$ \\ ${ }^{1}$ Dipartimento Scienze Mediche, Università degli Studi \\ di Milano, Fondazione IRCCS Ca' Granda, Ospedale \\ Maggiore Policlinico, Milano, Italy, ${ }^{2}$ Fondazione F.lli \\ Confalonieri, Dipartimento Scienze Mediche, Università \\ degli Studi di Milano, Fondazione IRCCS, Cà Granda \\ Ospedale Maggiore Policlinico, Milano, Italy, ${ }^{3}$ Dipartimento \\ di Medicina Preventiva Clinica e del Lavoro, U.O. Medicina \\ del Lavoro, Fondazione IRCCS Ca' Granda Ospedale \\ Maggiore Policlinico, Milano, Italy, ${ }^{4}$ Dipartimento di \\ Scienze Cliniche 'Luigi Sacco', Università degli Studi di \\ Milano, Milano, Italy \\ E-mail: fabrizia.bamonti@unimi.it
}

The negative effect of long term cigarette smoking on nutrition markers and oxidative status can be mitigated by supplementation.

A prospective randomized, double-blind, placebo-controlled study was made to evaluate the potential beneficial effects of a three months' supplementation with two different encapsulated formulas (Juice Plus ${ }^{\circledR}$, and Juice Plus ${ }^{\circledR}+$ Vineyard Blend), consisting primarily of mixed fruit and vegetable juice powder concentrate, on homocysteine (Hcy) metabolism and oxidative status.

One hundred and one apparently healthy (spirometric test) heavy smokers ( $>20$ cigarettes/day, duration $>10$ years; aged $47 \pm 8.7,54 \mathrm{M}$ ) were randomized into three groups: group P, placebo; group FV, fruit/ vegetables; group FVB, fruit/vegetables/berries. Plasma tHcy, serum vitamin B12 and holotranscobalamin (HoloTC, the biologically active form of B12), serum (s-Fol) and erythrocyte folate (ery-Fol) concentrations were determined by immunoenzymatic assay using the relevant commercial kits on automated analyzer AxSYM (Abbott Diagnostics, USA). Moreover, changes in oxidative-INDEX (Oxy-I, calculated on the basis of serum hydroperoxides and total anti-oxidant capacity measured by spectrophotometric method), oxidized low density lipoproteins levels (ox-LDL; ELISA method), malondialdehyde (MDA; gas chromatography-mass spectrometry method) in the free (fMDA), bound (bMDA) and total (tMDA $=\mathrm{fMDA}+\mathrm{bMDA})$ forms were evaluated.

Compared to placebo, both FB and FVB groups (mean compliance $85 \%$ ) showed, after supplementation, a significant increase in s-Fol and ery-Fol $(p<0.001)$, a significant decrease in tHcy $(p<0.001)$ concentrations and in Oxy-I $(\mathrm{p}<0.001)$, and, moreover, only in the $>95 \%$ compliant subjects, a significant decrease in ox-LDL $(p<0.05)$ and fMDA levels $(\mathrm{p}<0.01)$, and pulmonary improvement.

Both nutraceutical formulations provided bioavailable nutrition leading to significantly improved metabolic modifications compared to baseline values and to placebo. 


\section{Reference:}

Kawashima A et al. Asia Pac. J. Clin. Nutr. (2007);16:411-21.

6

Stability Evaluation to Formulate a Nutraceutical with Polyunsaturated Fatty Acids (PUFAs) and Oxidation-catalyzing Iron and Zinc

C. Freudiger ${ }^{1,2}$, B. Kriwet ${ }^{2}$, F. Müller ${ }^{2}$, E. Homberger ${ }^{2}$, S. Mühlebach ${ }^{1,3}$

${ }^{1}$ Institute of Clinical Pharmacology \& Toxicology,

Department of Pharmaceutical Sciences, University Basel, Basel, Switzerland, 2Vifor Ltd, Ettingen, Switzerland, ${ }^{3}$ Vifor Pharma, Glattbrugg, Switzerland

E-Mail: celine.freudiger@stud.unibas.ch

Introduction: For 'mental and cognitive health performance' EFSA (European Food Safety Authority) accepts RDA (recommended daily allowances) of Fe $(15 \mathrm{mg} / \mathrm{d})$ and $\mathrm{Zn}(10 \mathrm{mg} / \mathrm{d}) . \Omega-3$ PUFA are essential for brain development and recommended upon deficiency, during pregnancy and early childhood. Equazen ${ }^{\circledR}$, a marketed PUFA nutraceutical, demonstrated positive effects on working memory (learning skills) $(1,2)$. To check redox interactions of Fe, $\mathrm{Zn}$ and a vegetable antioxidant (AO) with PUFA a simple liquid formulation test was evaluated.

Methods: Tocopherol-stabilized PUFA triglycerides (DHA $\left(\mathrm{C}_{22: 5}\right)$ :EPA $\left(\mathrm{C}_{20: 5}\right)$ :GLA=9:3:1 [rel.conc.]) was assayed for peroxides $[\mathrm{PV}]$ and $\mathrm{p}$-anisidine $[\mathrm{AV}]$ for primary and secondary oxidation products (Ph.Eur.). The oil-mineral suspension was filled in brown snap cap vials. Different Fe(II)salts, a Fe(III)-hydroxy-polymaltose complex (Maltofer ${ }^{\circledR}$ ), inorganic and organic Zn salts in RDA were tested at $40^{\circ} \mathrm{C}$ and $75 \%$ rel. humidity over 4 weeks. Three samples per time point were centrifuged $(10 \mathrm{~min} 4000 \mathrm{rpm})$ and the supernatant analyzed. A plant-derived $\mathrm{AO}$, rich in phenols and rosmarinic acid, was checked for stabilizing. Saturated medium chain triglycerides $\left(\right.$ Miglyol $^{\circledR}$ ) served as blank.

Results: $\mathrm{PV} / \mathrm{AV}[\mathrm{mean} \pm \mathrm{SD}]$ at $40^{\circ} \mathrm{C} / 75 \%$ humidity; *exceeding $\mathrm{Ph}$. Eur. limits.

Blank: 0/0

Equazen $^{\circledR} \quad(\mathrm{t}=0): \quad 1.0 \pm 0.0 / 6.9 \pm 0.9 ; \quad(\mathrm{t}=4 \quad$ weeks $): \quad 38.7 \pm 0.2 * /$

$24.1 \pm 2.5$

Equazen ${ }^{\circledR}+\mathrm{Fe}$ sulfate or fumarate or Maltofer ${ }^{\circledR}(\mathrm{t}=4 \mathrm{w}): 18.2 * / 143 *$ or $25.6 * / 330^{*}$ or $27.5 * / 150 *$

Equazen ${ }^{\circledR}+\mathrm{Zn}$ sulfate or $\mathrm{Zn}$ lactate $(\mathrm{t}=4$ weeks $)$ : $19.0^{*} / 140.0^{*}$ or $11.0 * / 31.3 *$

Equazen $^{\circledR}+$ Maltofer $^{\circledR}+$ Zn lactate:

Without AO ( $\mathrm{t}=0): 1.2 \pm 0.0 / 2.1 \pm 0.3 ;(\mathrm{t}=4$ weeks): $20.8 \pm 0.1 * / 49.8 \pm$ $0.2^{*}$

With AO $(\mathrm{t}=0): 0.0 \pm 0.0 / 0.6 \pm 0.1 ;(\mathrm{t}=4$ weeks $): 16.3 \pm 0.1 * / 14.1$ $\pm 0.4$

\section{Conclusions:}

1. Ambient air and elevated temperatures oxidize liquid PUFA readily despite tocopherol content $(\mathrm{Ph}$.Eur. limits for PV $(<10)$ and/ or AV $(<30)$ exceeded within 4 weeks $)$.
2. Transformation of peroxides into secondary products is not conc.-correlated, but catalyzed by: $\mathrm{Fe}$ (III) $<\mathrm{Fe}$ (II); $\mathrm{Zn}$ lactate $<\mathrm{Zn}$ sulfate. The AO significantly increased the stability (PV and AV within the limits after 4 weeks stress test).

3. The proposed oxidation test in a liquid formulation is sensitive, reliable, non-expensive and assists a rational evaluation of PUFA formulations with potentially interacting pro-oxidatives.

\section{References:}

1. Richardson AJ, Montgomery P: The Oxford Durham study: a randomized controlled trial of dietary supplementation with fatty acids in children with developmental coordination disorder. Pediatrics. 2005; 115 (5):1360-1366.

2. Portwood M.M.: The role of dietary fatty acids in children's behaviour and learning. Nutrition and Health 2006; 18: 219-232.

7

\section{Natural Vegetarian Meal Replacement: Metabolic Modulation and Effects on Oxidative Stress}

R. De Giuseppe1, L. Vigna' ${ }^{2}$ C. Novembrino ${ }^{3}$, F. de Liso', D. Sommaruga 2 , A. Cossovich ${ }^{2}$, R. Maiavacca ${ }^{4}$, F. Bamonti

${ }^{1}$ Fondazione F.lli Confalonieri, Dip. Scienze Mediche, Università degli Studi di Milano, Fondazione IRCCS, Cà Granda Ospedale Maggiore Policlinico, Milano, Italy, ${ }^{2}$ Dipartimento Medicina Preventiva, UO Medicina del Lavoro I Fondazione IRCCS Ca' Granda - Ospedale Maggiore Policlinico, Milano, Italy, ${ }^{3}$ Dip. Scienze Mediche, Università degli Studi di Milano, Fondazione IRCCS, Cà Granda Ospedale Maggiore Policlinico, Milano, Italy, ${ }^{4}$ Laboratorio Patologia Clinica, Diparimento Area Servizi Diagnostici, Fondazione IRCCS Cà Granda Ospedale Maggiore Policlinico, Milano, Italy

E-mail: rachele.degiuseppe@unimi.it

Changes in lifestyle with short lunch breaks contributed to ever more frequently prescribed meal replacements in medical practice. GoJuvo $^{\circledR}$, a new commercial plant-based vegetarian meal, is composed by low glycemic index food. As very few data are available on GoJuvo metabolic effects, our aim was to verify if nutritional GoJuvo claims agreed with scientific tests by assessing nutritional parameters. Serum glucose, insulin and triglycerides concentrations were evaluated 3 times in 20 patients $\left(4 \mathrm{M} / 16 \mathrm{~F}\right.$, mean BMI $31.4 \mathrm{~kg} / \mathrm{m}^{2}$, aged $50.4 \pm 11.5$, enrolled at the Obesity and Work outpatients Clinic of the Milan Policlinico Hospital: T0 after overnight fasting; T1a 2 hours after GoJuvo administration ( $40 \mathrm{~g}$ in $300 \mathrm{ml}$ of plain water); T1b 2 hours after a standard Mediterranean meal (60\% carbohydrates, $25 \%$ lipids, $15 \%$ proteins). In 10 women (aged $54 \pm 8.4$ ) glycemic status and lipid panel were also evaluated three months after taking GoJuvo as meal replacement (T2).

Results: expressed as mean $\pm \mathrm{SD}$ and, in brackets, mean delta values percentage of parameters' changes.

Glucose $(\mathrm{mg} / \mathrm{dL}): \mathrm{T} 0=91.6 \pm 9.2, \mathrm{~T} 1 \mathrm{a}=87.0 \pm 10.7(-4.3 \%), \mathrm{T} 1 \mathrm{~b}=94.9$ $\pm 23.1(+4.4 \%)$

Triglycerides $(\mathrm{mg} / \mathrm{dL}): \quad \mathrm{T} 0=96.8 \pm 8.1, \quad \mathrm{~T} 1 \mathrm{a}=89.9 \pm 36.1(-5.8 \%)$, $\mathrm{T} 1 \mathrm{~b}=119 \pm 48.8(+37.6 \%)$ 
Insulin $(\mathrm{mg} / \mathrm{dL}): \mathrm{T} 0=10.6 \pm 7.1, \mathrm{~T} 1 \mathrm{a}=11.0 \pm 13.4(+2.1 \%), \mathrm{T} 1 \mathrm{~b}=49.0$ $\pm 60.9(+306 \%)$

The increase in glycaemia, insulin and triglycerides mean delta values percentage was lower after GoJuvo administration than after the Mediterranean meal. At T2 no significant changes were found in glycemic status, but HDL-cholesterol concentrations were significantly higher than at T0 $(58.3 \pm 13.6$ vs $50.0 \pm 13.5 \mathrm{mg} / \mathrm{dL} ; \mathrm{p}=0.015)$ and triglycerides concentrations were lower at T2 than at T0, though not significant $(76.3 \pm 16.1$ vs $94.1 \pm 38.0 \mathrm{mg} / \mathrm{dL} ; \mathrm{p}=0.09)$. Our data suggest that GoJuvo metabolic modulations are better than post-prandium ones due to $60 \%$ of integral rice contained in $40 \mathrm{~g}$ of GoJuvo, mixed with other vegetables and fruit. GoJuvo prolonged use seems to improve the lipid panel.

\section{Reference:}

1. Konig D et al. Ann Nutr Metab (2008); 52:74-8.

8

\section{Protective Effect of Propill, a Quercetin/ Zinc-Based Food Supplement, on Oral Contraception-Induced Oxidative Stress}

\author{
D. De Groote1, A. Pintiaux², C. Sayada ${ }^{1}$, F. Chantraine², \\ A. Beliard', M. Nisolle', C. Gerday², B. Balteau², \\ A. Mukaneza', J.M. Foidart ${ }^{2}$ \\ ${ }^{1}$ PROBIOX SA, Belgium, ${ }^{2}$ Département de Gynécologie et \\ Obstétrique, Université de Liège, Liège, Belgium \\ E-mail:d.degroote@probiox.com
}

We previously showed that oral contraception (OC) with ethinylestradiol and drospirenone is associated with an oxidative stress (OS) status characterized by high levels of lipid peroxides (LOOH) and oxidized LDLs (oxLDL), and by an unfavourable copper to zinc ratio (1). Because such OS profile could be associated with increased cardiovascular risk, we prepared a specific dietary supplement (ProPillTM) susceptible to reduce the levels of OS in women under OC.

A double blind randomized placebo controlled study was conducted to validate the effect of ProPill ${ }^{\mathrm{TM}}$ on oxidative stress. 50 women under OC with ethinylestradiol and drospirenone were randomized to receive ProPillTM $(n=15)$, placebo $(n=15)$, quercetin alone $(n=10)$ or zinc alone $(n=10)$. The different investigational products were taken during four consecutive menstrual cycles. The levels of $\mathrm{LOOH}$, oxLDL, $\mathrm{Cu}$ and $\mathrm{Zn}$, and the expression of 200 genes involved in oxidative stress pathways were assessed at different time points. No significant adverse effects were recorded during the study.

Results showed a significant reduction of the $\mathrm{Cu} / \mathrm{Zn}$ ratio during the ProPill ${ }^{\mathrm{TM}}$ treatments. The reduction was statistically significant compared to placebo and to quercetin alone. A significant decrease of oxLDL was only observed during the ProPill ${ }^{\mathrm{TM}}$ treatment and the decrease was statistically significant compared to placebo. In addition, the microarray analysis revealed 44 genes to be significantly differentially expressed in the group treated with ProPill ${ }^{\mathrm{TM}}$ compared to the placebo group. The observed gene profile suggests that the treatment with ProPill ${ }^{\mathrm{TM}}$ regulates the expression of genes involved in different biological pathways.
In conclusion, the synergistically and complementary biological effects of quercetin and zinc in the ProPillTM formulae and the absence of significant adverse events observed during the study, suggest that ProPill ${ }^{\mathrm{TM}}$ could efficiently protect women from some secondary effects of oral contraception.

\section{Reference:}

D. De Groote et al. Contraception 80 (2009) 187-193.

\section{9 \\ Supplementation with Encapsulated Vegetable and Fruit Juice Powder Concentrate Improves Microcirculation and Ultrastructure in Skin}

U. Heinrich, B. Garbe, M. Wiebusch, H. Tronnier

Institute for Experimental Dermatology, Dermatronnier, University of Witten/Herdecke, Witten/Herdecke, Germany

E-mail: ulrike.heinrich@uni-wh.de

The objective of the study was to determine changes in skin physiological parameters during the intake of vegetable and fruit juice powder concentrate (JPC) vs. placebo capsules. The detection of microcirculation, hydration, transepidermal water loss, ultrastructure, i.e. skin thickness and density were determined during the study.

Microcirculation measurements were performed by the $\mathrm{O} 2 \mathrm{C}$ device (Lea Instruments, Giessen, Germany), hydration measurements with the Corneometer CM 825 prior to and during the study. Transepidermal water loss was measured with the Tewameter (Courage \& Khazaka Electronics, Cologne, Germany). Analyses of skin thickness and density were made by ultrasound measurements (Dermascan C, Cortex Technology).

52 female volunteers took part in the study, from 40 to 65 years in age, complying with all inclusion criteria. The study was performed as a randomized, placebo-controlled, double-blind study. All tests were performed prior to first intake of the described capsules and after 6 and 12 weeks.

All test results were statistically analyzed. Descriptive statistics and pre-post differences were calculated and each combination was compared. Percentage changes and $\mathrm{p}$-values were determined at all measuring points. The following results were obtained in this study:

1. Significant improvement of microcirculation by $36 \%$ (flow $1 \mathrm{~mm}$ depth) could be shown after 12 week supplementation with the JPC capsules.

2. Significant improvement of the skin thickness by $6 \%$ and skin density by $17 \%$, measured by ultrasound (B-Scan) was detected in the JPC group

3. Significant increase of skin hydration by $9 \%$ as well as a decrease of TEWL by $4 \%$ by the treatment after 12 weeks could be shown. This constitutes an improvement of the barrier function of the skin.

Microcirculation and ultrastructure of the skin could be improved. Further skin physiological parameters like skin hydration, barrier function were positively influenced. The placebo group showed only minor or no positive effects. 


\section{Reference:}

Heinrich U, Tronnier H, Stahl W, Bejot M, Maurette JP. Antioxidant Supplements Improve Parameters Related to Skin Structure in Humans. Skin Pharmacol Physiol 2006;19:224-231.

10

\section{Evaluation of Oxidative Status in Osteopenic and Osteoporotic Women}

\author{
F. de Liso', C. Novembrino ${ }^{2}$, R. De Giuseppe ${ }^{1}$, F. Bamonti ${ }^{2}$ \\ ${ }^{1}$ Fondazione F.lli Confalonieri, Dip. Scienze Mediche, \\ Università degli Studi di Milano, Fondazione IRCCS, Cà \\ Granda Ospedale Maggiore Policlinico, Milano, Italy, \\ ${ }^{2}$ Dip. Scienze Mediche, Università degli Studi di Milano, \\ Fondazione IRCCS, Cà Granda Ospedale Maggiore \\ Policlinico, Milano, Italy \\ E-mail: federica.deliso@gmail.com
}

Background: Osteopenia is a condition where bone mineral density is lower than normal and is considered to be a precursor of osteoporosis, a systemic skeletal disease characterized by decreased bone mineral density causing an increase in bone fragility and susceptibility to fractures.

After menopause, bone turnover acceleration induces an imbalance between bone resorption and formation, leading to final postmenopausal bone loss.

It has been suggested that oxidative stress, as a consequence of an imbalance between oxidants and antioxidants, is associated with osteoporosis pathogenesis.

Aim: Evaluating oxidative status in osteopenic and osteoporotic women.

Methods: Thirty consecutive menopausal and post-menopausal female subjects (aged $63 \pm 8.88$; mean BMI $24.91 \pm 3.88 \mathrm{Kg} / \mathrm{m}^{2}$ ) were selected by using ultrasonographic examination of bone tissue (DBM Sonic Bone Profiler, IGEA, Italy), divided into: Group A: 16 osteopenic subjects (aged 59.62 \pm 9.12 ; mean BMI $25.03 \pm 4.50 \mathrm{Kg} /$ $\mathrm{m}^{2}$ ) and Group B: 14 osteoporotic subjects (aged 66.85 \pm 7.06 ; mean BMI $24.7 \pm 3.11 \mathrm{Kg} / \mathrm{m}^{2}$ ) and evaluated for serum Reactive Oxygen Species concentrations (ROS) and Total Antioxidant Capacity (TAC) both by spectrophotometric methods (Diacron International, Italy).

Results: ROS concentrations were significantly higher in group A than in group B $(420 \pm 46.79$ UCarr vs $365 \pm 47.68 ; p=0.003)$. No significant difference was found between the two groups as regards TAC.

Conclusions: Our preliminary results showed an increased oxidative stress condition in osteopenic subjects, due to osteoclastic activity which is probably higher in osteopenia than in osteoporosis.

A balance between oxidants and antioxidants is important for maintaining a correct equilibrium between osteoblast and osteoclast activities and, therefore, regulating bone resorption.

\section{Reference:}

Banfi G. et al. Clin Chem Lab Med (2008); 46:1550-5.

\section{1}

\section{Oral Administration of Neutral Lactase Significantly Reduces Symptoms of Lactose Intolerance}

\author{
A. Missbichler'1, Lucas Fraiss/2, Roland Leitner ${ }^{3}$ \\ ${ }^{1}$ Sciotec Diagnostic Technologies GmbH, Tulln, Austria \\ ${ }^{2}$ University of applied Sciences Wr. Neustadt, Department \\ of Biotechnical Processes, Tulln, Austria ${ }^{3}$ Department \\ of Medical Biochemistry, Medical University of Vienna, \\ Vienna, Austria \\ E-mail: albert@sciotec.at
}

Lactose intolerance is the insufficient ability to digest lactose, a sugar commonly found in dairy products. It is caused by a deficiency of the enzyme lactase (EC 3.2.1.32) in the small intestine. Lactase deficiency normally develops slowly after adolescence or adulthood. Typical symptoms are abdominal pain, bloating, diarrhea and nausea.

Here we present a novel formulation using neutral lactase for replenishing the lack of enzyme in the small intestine. The enzyme is produced in klyveromyces lactis, a yeast well known from cheese production. Based on our know-how with Diamine Oxidase and Xylose Isomerase the enzyme is embedded and stabilized in small pellets of $1 \mathrm{~mm}$ diameter, which are enteric coated with shellac thus protecting lactase from gastric juice. Because of the small size the pellets pass the stomach within $15 \mathrm{~min}$ to reach the small intestine. In the neutral surroundings the pellets disintegrate and release the activity of the neutral lactase. The activity of the enzyme persists for several hours and ensures effective degradation of lactose.

First results of an observational study based on data obtained from 38 volunteers support the expected efficacy of the novel formulation: a total reduction of $65 \%$ of symptoms was reported. These data confirm the high performance and effectivity of enzyme supplementation in the small intestine using an enteric coated formulation.

\section{References:}

Heyman MB: Lactose intolerance in infants, children, and adolescents. Pediatrics. 118:1279, 2006.

Itan Y, Powell A, Beaumont MA, Burger J, Thomas MG: The origins of lactase persistence in Europe. PLoS Comput Biol. 5(8):e1000491, 2009. 


\section{2}

\section{Determinants of Oxidative Stress Related to Gender: Relevance of Age and Smoking Habits}

\author{
C. Novembrino ${ }^{1}$, F. Bamonti ${ }^{1}$, R. De Giuseppe ${ }^{2}$, F. de Liso², \\ S. Maffei ${ }^{3}$, A. Mercuri ${ }^{3}$, C. Vassalle 3 \\ ${ }^{1}$ Dip. Scienze Mediche, Università degli Studi di Milano, \\ Fondazione IRCCS Ca' Granda, Ospedale Maggiore, \\ Milano, Italy, ${ }^{2}$ Fondazione F.lli Confalonieri, Dip. Scienze \\ Mediche, Università degli Studi di Milano, Fondazione \\ IRCCS, Cà Granda Ospedale Maggiore Policlinico, Milano, \\ Italy, ${ }^{3}$ Fondazione G. Monasterio CNR-Regione Toscana \\ and Institute of Clinical Physiology-CNR, Pisa, Italy \\ E-mail: cristina.novembrino@unimi.it
}

Magnitude and major causes of oxidative stress may be different between men and women. Gender effects on oxidative status have been addressed in clinical studies only to a limited extent with controversial results.

Aim: In the present study, we aimed to determine whether any gender-related difference exists concerning oxidative stress in a population of 332 subjects of both sexes, in a wide age-range, with and without cigarette smoking habit.

Methods: The Oxidative-INDEX was calculated after evaluation of serum hydroperoxides (ROMs) and total antioxidant capacity (OXY) by means of the relevant commercial kits (d-ROMs and Oxy-adsorbent Tests, Diacron, Italy) subtracting the OXY standardized variable from the ROMs standardized variable, and expressed as arbitrary units (AU).

Results: The Oxidative-INDEX was higher in women than in men $(\mathrm{p}<0.001)$, in smokers $(\mathrm{p}<0.01)$ than in non-smokers, and correlated with number of cigarettes $(p<0.01)$, age $(p<0.001)$ and postmenopausal status $(\mathrm{p}<0.001)$. The multivariate analysis identified age, high blood pressure and smoking habit as factors independently associated with the Oxidative-INDEX in men, whereas cigarette smoking and age represented the independent risk factors for an elevated oxidative stress status in women.

Conclusion: Gender-based differences in oxidative stress levels may provide a biochemical basis for the epidemiologic differences in the disease susceptibility between sexes, and suggest different strategies for risk assessment, diagnosis and treatment specifically targeted to men and women.

\section{Reference:}

C. Vassalle et al. Clin Biochem (2009); 42:739-41.

\section{3}

\section{Changes in Lipid Peroxidation Products and} Antioxidant Activities in Anemic Women Before and After Oral Iron Supplementation

\author{
A. Tiwari ${ }^{1}$, A.A. Mahdi ${ }^{1}$, F. Mahdi² \\ ${ }^{1}$ Department of Biochemistry, C.S.M. Medical University, \\ Lucknow, U.P., India, 'Department of Biochemistry, Era's \\ Lucknow Medical College \& Hospital, Lucknow, U.P., India \\ E-mail:manikgmc@gmail.com
}

Background \& Aims: Iron deficiency anemia (IDA) is one of the most common nutritional disorders worldwide, affecting all ages (1). Oral iron supplementation is a common used strategy to meet the increased requirements of risk groups (2). On going through the available literature there seem to be limited and contradictory data available on oxidative stress and antioxidant defense parameters in iron deficient anemic women and also on the effect of iron supplementation on antioxidant status. The present study was designed to evaluate the lipid peroxidation products and the antioxidative enzymes in iron deficient anemic women and their response to oral iron supplementation.

Methods: Iron and folic acid supplementation were given to 240 anemic women $($ mild $=100$, moderate $=100$ and severe $=40)$ and 100 age matched placebo treated (100 mg cane sugar) non-anemic controls daily for 100 days. All parameters were estimated as per standard protocols.

Results: Blood indices along with antioxidant enzymes, namely catalase, superoxide dismutase, glutathione reductase, total antioxidant capacity and ferritin levels were found significantly increased $(p<0.01)$ in anemic women after treatment. However, glutathione peroxidase and transferring receptor levels were found significantly decreased in all treated groups. Lipid peroxide levels, protein carbonyl contents, conjugated dienes and lipid hydroperoxide levels were found significantly increased $(\mathrm{p}<0.01)$ after oral iron supplementation groups. Moreover, undesirable side effects of iron supplementation were observed maximum in mild as compared with moderate and severe anemic groups.

Conclusion: On the basis of our results, it may concluded that $100 \mathrm{mg}$ iron and $500 \mu \mathrm{g}$ folate/day helps to recover blood indices, however, it also enhances the oxidative stress. Therefore, more studies are needed to determine the exact interventational dose, added with antioxidants, which not only help to increase $\mathrm{Hb}$ levels but also maintains oxidative stress profiles during iron supplementation.

\section{References:}

1. World Health Organization. Iron Deficiency: Assessment, Prevention and Control. Geneva, Switzerland: World Health Organization, 2001.

2. Galaris D, Pantopoulos K. Oxidative stress and iron homeostasis: mechanistic and health spects. Crit Rev Clin Lab Sci 2008;45:1-23. 


\section{Author Index}

\section{Anals of \\ utrition\& Metabolism}

Balteau, B. 8

Bamonti, F. 5, 7, 10, 12

Beliard, A. 8

Chantraine, F. 8

Cighetti, G. 5

Cossovich, A. 7

De Giuseppe, R. 5, 7, 10, 12

De Groote, D. 8

de Liso, F. 5, 7, 10, 12

Foidart, J.M. 8

Fraissl, L. 11

Freudiger, C. 6

Numbers refer to abstract numbers

Garbe, B. 9

Gerday, C. 8

Heinrich, U. 1, 9

Homberger, E. 6

Huber, J.C. 3

Krafft, A. 4

Kriwet, B. 6

Leitner, R. 11

Maffei, S. 12

Mahdi, A.A. 13

Mahdi, F. 13
Maiavacca, R. 7

Mercuri, A. 12

Missbichler, A. 11

Mühlebach, S. 6

Mukaneza, A. 8

Müller, F. 6

Nisolle, M. 8

Novembrino, C. 5, 7, 10, 12

Ogundipe, E. 2

Pellegatta, M. 5

Pintiaux, A. 8
Sayada, C. 8

Sommaruga, D. 7

Tiwari, A. 13

Tronnier, H. 9

Vassalle, C. 12

Vigna, L. 5, 7

Wiebusch, M. 9

\section{KARGER}

(C) 2011 S. Karger AG, Basel

Fax +41 613061234 